\title{
Relevancia del monitoreo continuo de glucosa en la práctica clínica: revisión de la evidencia
}

\author{
Ana María Gómez ${ }^{1,2 A}$, Diana Cristina Henao Carrillo ${ }^{1,2 A}$, Lucía Taboada ${ }^{1,2 A}$, \\ Martín Rebolledo Del Toro ${ }^{1}$
}

${ }^{1}$ Pontificia Universidad Javeriana, Facultad de Medicina, Bogotá, Colombia.

${ }^{2}$ Hospital Universitario San Ignacio, Bogotá, Colombia.

${ }^{A}$ Unidad de Endocrinología

Autor de Contacto

Ana María Gómez, MD

amgomezm5@gmail.com

Fecha de recepción: 27/04/2018

Fecha de aceptación: 29/05/2018

\section{Resumen}

$\mathrm{E}$ I monitoreo continuo de glucosa (MCG) es una herramienta que permite evaluar el control glucémico, la variabilidad glucémica y la detección de episodios de hipoglucemia asintomática y nocturna. Actualmente disponemos de dispositivos de última generación que son más precisos y sensibles para la detección de hipoglucemia, lo cual ha permitido el desarrollo de algoritmos predictores con el fin de prevenir dichos eventos, ya sea utilizando alarmas para que el paciente intervenga o suspendiendo la infusión de insulina. Esta tecnología se encuentra disponible en Colombia y ha demostrado ser una alternativa segura y efectiva en el tratamiento de pacientes diabéticos con alto riesgo de hipoglucemia severa y otras poblaciones especiales como niños y mujeres embarazadas. El objetivo de esta revisión es resumir la evidencia clínica relevante, ventajas y desventajas de esta terapia, así como el impacto clínico del uso del MCG en tiempo real (MCG-TR) en pacientes en tratamiento con múltiples dosis o sistemas de infusión subcutánea de insulina.

\section{Summary}

Continuous glucose monitoring (CGM) is a tool that allows the evaluation of glycemic control, glycemic variability and detection of episodes of asymptomatic and nocturnal hypoglycemia. Currently we have state-of-the-art devices that are more precise and sensitive for the detection of hypoglycemia, which has allowed the development of predictive algorithms in order to prevent such events either by using alarms for the patient to intervene or by stopping the infusion of insulin. This

technology is available in Colombia and has proven to be a safe and effective alternative in the treatment of diabetic patients with a high risk of severe hypoglycemia and other special populations such as children and pregnant women. The objective of this review is to summarize the relevant clinical evidence, advantages and disadvantages of this therapy as well as the clinical impact of the use of the MCG in real time (MCG-TR) in patients in treatment with multiple doses of insulin or insulin infusion systems.

\section{Introducción}

La vigilancia del control glucémico es primordial en el manejo del paciente con diabetes. Cohortes de seguimiento a largo plazo describieron la asociación entre niveles de hemoglobina glucosilada (HbA1c) y la incidencia de complicaciones microvasculares ${ }^{(1,2)}$. Aunque la HbA1c persiste como marcador de referencia para evaluar el control glucémico y es un indicador validado como factor de riesgo para complicaciones, tiene varias limitaciones, entre ellas: 1) provee únicamente el promedio de glucosa durante los últimos 2 a 3 meses; 2 ) no detecta hipo o hiperglucemia en el día a día; 3 ) no es confiable en pacientes con anemia o hemoglobinopatías ${ }^{(3)}$.

El automonitoreo de glucosa capilar (Self-monitoring of the blood glucose, SMBG) se considera como el método de oro para la evaluación del control glucémico en el día a día del paciente diabético; sin embargo, la información brindada por una glucometría capilar está limitada a la medición de glucosa preprandial, las mediciones adicionales son definidas por el paciente, lo que dificulta la detección de hipoglucemias asintomáticas y nocturnas ${ }^{(4)}$ y su impacto en el control metabólico depende del número de mediciones ${ }^{(5-7)}$.

El monitoreo continuo de glucosa (MCG) es una herramienta valiosa disponible en el mundo desde 1999, se clasifica en profesional o retrospectivo y en tiempo real (MCG-RT) el cual, utiliza algoritmos capaces de anticipar episodios de hipo e hiperglucemia basados en la tendencia de la glucosa intersticial e informan al paciente de los valores y las tendencias, permitiéndole tomar acción para evitar hiper e hipoglucemia ${ }^{(8)}$. Existe una gran variedad de dispositivos que utilizan un sensor transcutáneo que registra los niveles de glucosa intersti- 
cial 24 horas al día, generando información adicional como la tasa de cambio de niveles de glucosa en relación al tiempo ${ }^{(9)}$, tiempo en rangos y detección y duración de los episodios de hipo e hiperglucemia ${ }^{(3)}$.

\section{Exactitud clínica y numérica de los dispositivos de MCG}

Dado que estos dispositivos miden los niveles de glucosa intersticial, es importante saber qué tan precisos son los datos obtenidos por sistemas de MCG, comparados con las lecturas de automonitoreo capilar. Las mediciones en este compartimento difieren en tiempo y magnitud de los valores de glucemia correspondientes y las variaciones son mayores cuando hay cambios rápidos en la concentración de glucosa plasmática ${ }^{(7,10)}$. Debido al retraso fisiológico e instrumental, esta diferencia es de 13,5 minutos, aproximadamente ${ }^{(11,12)}$.

Hay diferentes formas de evaluar la precisión de los datos obtenidos ${ }^{(13-15)}$. El valor de la diferencia absoluta relativa (mean absolute relative difference [MARD\%]) entre glucosa capilar y la glucosa intersticial (método de referencia vs. intersticial dividido por el valor de referencia) expresado como un porcentaje o una media de la diferencia absoluta, el cual corresponde a la precisión numérica ${ }^{(15)}$. El MARD\% de los dispositivos disponibles en nuestro país para valores en el rango son de 13,2\% \pm 10,9\% (Abbott Diabetes Care, Alameda, CA, USA) y $21,4 \% \pm 17,6 \%$ (Medtronic, Northridge, CA, USA) en condiciones de la vida real ${ }^{(16)}$ cumpliendo la norma ISO (Organización Internacional de Estandarización) 15197:2013, la cual establece que el MARD\% debe ser menor del $15 \%{ }^{(12,13)}$. Los sensores de nueva generación con el objetivo de mejorar la exactitud de las mediciones, especialmente en hipoglucemia, tienen mayor precisión (MARD\% $<10 \%)^{(9)}$, lo cual reduce la diferencia entre las mediciones de glucosa intersticial y capilar, y aumenta la sensibilidad para la detección de hipoglucemia( ${ }^{(7,17)}$.

Otra forma de evaluar la precisión es a través de métodos de análisis por tendencias clínicas como el Consensus Error Grid (CEG) ${ }^{(14,15)}$, cuya fortaleza yace en que mide la seguridad y eficacia de la información para tomar decisiones clínicas e identificar la frecuencia de errores y el desempeño del dispositivo de acuerdo con zonas A, B, C, D y E; porcentajes mayores en zona $\mathrm{A}$ o $\mathrm{A}+\mathrm{B}$, indican mejor desempeño ${ }^{(13,15)}$.

La precisión de los dispositivos también depende del uso de un método de calibración reproducible y fiable. Existen dos tipos de calibración: el de fábrica y el que realiza el paciente $^{(18)}$. En la primera, el sensor es calibrado en la fábrica y por lo tanto no requiere de glucometrías capilares por parte del usuario ${ }^{(18)}$. En el segundo tipo, los dispositivos requieren calibración con glucosa capilar, la cual debe realizarse una vez implantado el sensor y su frecuencia varía entre 2 y 3 glucometrías diarias ${ }^{(19)}$.

\section{Características de los dispositivos para MCG disponibles en Colombia}

La tabla 1 resume los diferentes dispositivos disponibles en Colombia. El FreeStyle Libre Flash Glucose Monitoring System ${ }^{\circledR}$ utiliza un sensor calibrado en fábrica, que permite mediciones de glucosa intersticial con una frecuencia determinada por el número de veces que el paciente realice el "escaneo" con el lector; sin embargo, no emite alarmas que indiquen al paciente realizar acciones específicas ${ }^{(20)}$.

El iPRO2 permite realizar MCG retrospectivo. La precisión del sistema iPro2 se evaluó in vitro calibrando los sensores en soluciones con concentraciones de glucosa conocidas (medidas utilizando un analizador de glucosa de Yellow Springs Instrument, YSI), repitiendo la medición (al menos 10 veces) en soluciones con concentraciones dentro del rango de funcionamiento. Este estudio está incluido en el aplicativo MIPRES y provee información más completa para la toma de decisiones, permitiendo la detección de excursiones glucémicas, evaluación de variabilidad glucémica y ajustes de la terapia hipoglucemiante de acuerdo con patrones de glucosa. En estudios en mujeres embarazadas con diagnóstico de diabetes han demostrado mejoría del control metabólico.

En Colombia disponemos de dispositivos que utilizan CMGTR integrados a sistemas de infusión subcutánea o Sensor Augmented Insulin Pump Therapy (SAPT), como Paradigm ${ }^{\circledR} \mathrm{Veo}^{\mathrm{TM}}$ y MiniMed ${ }^{\circledR}$ 640G with SmartGuard ${ }^{\circledR}$ (Medtronic, Northridge, CA, USA ${ }^{(9,12)}$. El primer dispositivo cuenta con la función de suspensión en hipoglucemia o Low Glucose Suspend (LGS), y el segundo utiliza un algoritmo que suspende la infusión de insulina incluso antes de la hipoglucemia ${ }^{(12)}$. Este tipo de terapia se encuentra cubierta por el Plan de Beneficios en Salud (PBS) con diferentes centros especializados para el tratamiento de los usuarios de estos dispositivos.

\section{Utilidad del MCG en la práctica clínica}

\section{Eficacia: disminución de HbA1c}

La tabla 2 resume los estudios de MCG -TR con reducción de la HbA1c como objetivo primario comparado con SBMG ( $\geq$ 4 mediciones de glucosa capilar en el día) ${ }^{(6,7)}$.

Estudios como el JDRF (Juvenile Diabetes Research Foundation), que utilizó diferentes dispositivos y el STAR 3 compararon el uso de MCG-TR e infusión continua de insulina subcutánea vs. SMBG y múltiples dosis de insulina (MDI) en pacientes con DM1, reportando una reducción significativa de la HbA1c en los usuarios de MCG-TR ${ }^{(5,21)}$. Estudios similares han demostrado que la adición del MCG-TR a MDI comparado con SMBG lleva a un mejor control glucémico ${ }^{(22,23)}$.

El estudio RealTrend fue un ensayo clínico controlado que comparó la eficacia de la terapia con infusión continua de insulina subcutánea MCG vs. SMBG, demostrando que el uso del sensor por más del 70\% del tiempo, redujo la HbA1c en 
Tabla 1. Dispositivos disponibles en el mercado

\begin{tabular}{|c|c|c|c|c|c|c|c|c|c|c|}
\hline & Dispositivo & Transmisor & Sensor & $\begin{array}{c}\text { Tiempo } \\
\text { real }\end{array}$ & SAPT & $\begin{array}{c}\text { Precisión } \\
\text { (MARD, } \\
\% \text { ) }\end{array}$ & $\begin{array}{c}\text { Calibración } \\
\text { (n/días) }\end{array}$ & $\begin{array}{l}\text { Vida } \\
\text { útil del } \\
\text { sensor }\end{array}$ & $\begin{array}{c}\text { Disponible } \\
\text { en } \\
\text { Colombia }\end{array}$ & Observaciones \\
\hline \multirow{4}{*}{ 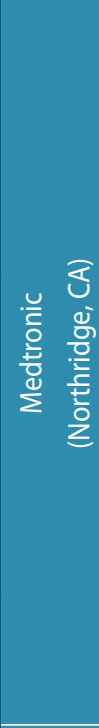 } & $\begin{array}{c}\text { Paradigm }^{\circledast} \\
\text { Veo }^{T M *}\end{array}$ & Minilink & Enlite & Sí & Sí & 13,6 & 3 & 6 & Sí & $\begin{array}{l}\text { Se suspende en } \\
\text { hipoglucemia. }\end{array}$ \\
\hline & $\begin{array}{c}\text { MiniMed }^{\circledR} \\
\text { 640G } \\
\text { SmartGuard }^{\circledR *}\end{array}$ & $\begin{array}{c}\text { Guardian } 2 \\
\text { link }\end{array}$ & Enlite & Sí & Sí & 14,2 & 3 & 6 & Sí & $\begin{array}{l}\text { Se suspende } \\
\text { previo a } \\
\text { hipoglucemia. }\end{array}$ \\
\hline & $\begin{array}{c}\text { MiniMed }^{\oplus} \\
\text { 670G } \\
\text { SmartGuard } \\
\text { HCL }\end{array}$ & $\begin{array}{c}\text { Guardian } 3 \\
\text { link }\end{array}$ & Enlite & Sí & Sí & 9,64 & 3 & 7 & No & $\begin{array}{l}\text { Aprobado por FDA } \\
\text { en > } 14 \text { años. Se } \\
\text { suspende previo a } \\
\text { hipoglucemia. En } \\
\text { modo automático } \\
\text { ajusta dosis de } \\
\text { insulina para evitar } \\
\text { hiperglucemias. }\end{array}$ \\
\hline & $\begin{array}{c}\text { iPro }^{\oplus} \\
\text { Professional } \\
\text { CGM system* }\end{array}$ & Minilink & $\begin{array}{l}\text { Enlite } \\
\text { and Soft- } \\
\text { sensor }\end{array}$ & No & No & 11 & 3 & 6 & Sí & \\
\hline \multirow{2}{*}{ 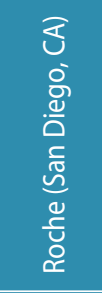 } & $\begin{array}{c}\text { Dexcom }^{\circledR} \mathrm{G} 4 \\
\text { PLATINUM } \\
\text { CGM }^{*}\end{array}$ & Dexcom G4 & $\begin{array}{l}\text { Dexcom } \\
\text { G4 }\end{array}$ & Sí & No & $\begin{array}{l}A: 13 \\
N: 15\end{array}$ & 2 & 7 & No & $\begin{array}{l}\text { Aprobado en } \\
\text { niños mayores de } \\
2 \text { años. }\end{array}$ \\
\hline & $\begin{array}{l}\text { Dexcom G5TM } \\
\text { Movile } \mathrm{CGM}^{\mathrm{TM}}\end{array}$ & Dexcom G5 & $\begin{array}{l}\text { Dexcom } \\
\text { G5 }\end{array}$ & Sí & No & $\begin{array}{l}A: 9 \\
N: 10\end{array}$ & 2 & 7 & No & $\begin{array}{l}\text { Aprobado por } \\
\text { FDA, en personas } \\
\text { mayores de } 16 \\
\text { años. }\end{array}$ \\
\hline \multirow{3}{*}{ 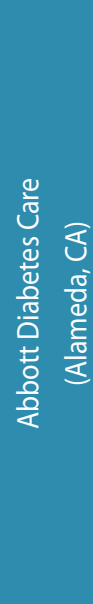 } & $\begin{array}{l}\text { FreeStyle Libre } \\
\text { Flash glucose } \\
\text { monitoring } \\
\text { system** }\end{array}$ & - & $\begin{array}{l}\text { FreeStyle } \\
\text { Libre } \\
\text { sensor }\end{array}$ & No & No & 10 & No & 14 & Sí & $\begin{array}{l}\text { Permite medir la } \\
\text { glucosa intersticial, } \\
\text { el número de } \\
\text { veces que el } \\
\text { paciente use el } \\
\text { lector. Sin alarmas. }\end{array}$ \\
\hline & $\begin{array}{c}\text { FreeStyle } \\
\text { Navigator II } \\
\text { CGM system }^{* *}\end{array}$ & Transmisor & $\begin{array}{l}\text { System } \\
\text { kit } \\
\text { (receptor, } \\
\text { trasmisor } \\
\text { y } \\
\text { cargador) }\end{array}$ & Sí & No & 12,3 & $5^{* *}$ & 5 & No & \\
\hline & $\begin{array}{c}\text { FreeStyle Libre } \\
\text { Pro }\end{array}$ & - & $\begin{array}{l}\text { FreeStyle } \\
\text { Libre } \\
\text { sensor }\end{array}$ & No & No & 11,1 & No & 14 & No & \\
\hline
\end{tabular}

MARD\% (diferencia absoluta media relativa), A: adultos, N: niños, SAPT: terapia integrada a sistema de monitoreo continuo en tiempo real. Adaptado de Gómez AM, Carrillo DCH, Velandia OMM. Devices for continuous monitoring of glucose: Update in technology. Med Devices Evid Res. 2017;10:215-24.

*Los dispositivos de Medtronic y Dexcom la tendencia de la glucosa intersticial es medida cada 5 minutos. (6)

**FreeStyle Navigator II CGM system require 4 calibraciones el primer día, 1 calibración al tercer día. En los dispositivos de Abbott, las lecturas de la glucosa intersticial son generadas cada minuto.(8) Datos tomados del manual de usuario de Abbott FreeStyle Navigator II.

$-0,41 \%(\mathrm{p}<0,004)^{(5,12,21)}$; hallazgos similares se han descrito en otros estudios, donde el uso del sensor al menos seis días por semana, independientemente del grupo de edad, se asoció con una reducción promedio de $\mathrm{HbA} 1 \mathrm{c}$ de $-0,5 \%{ }^{(21)}$, estableciendo una relación directamente proporcional entre el tiempo de uso del sensor y la reducción de la HbA1c, la cual es significativa con el uso del sensor por un tiempo mayor al $40 \%$ y se duplica en los sujetos que lo utilizaban más del $80 \%$ del tiempo ${ }^{(5)}$. 


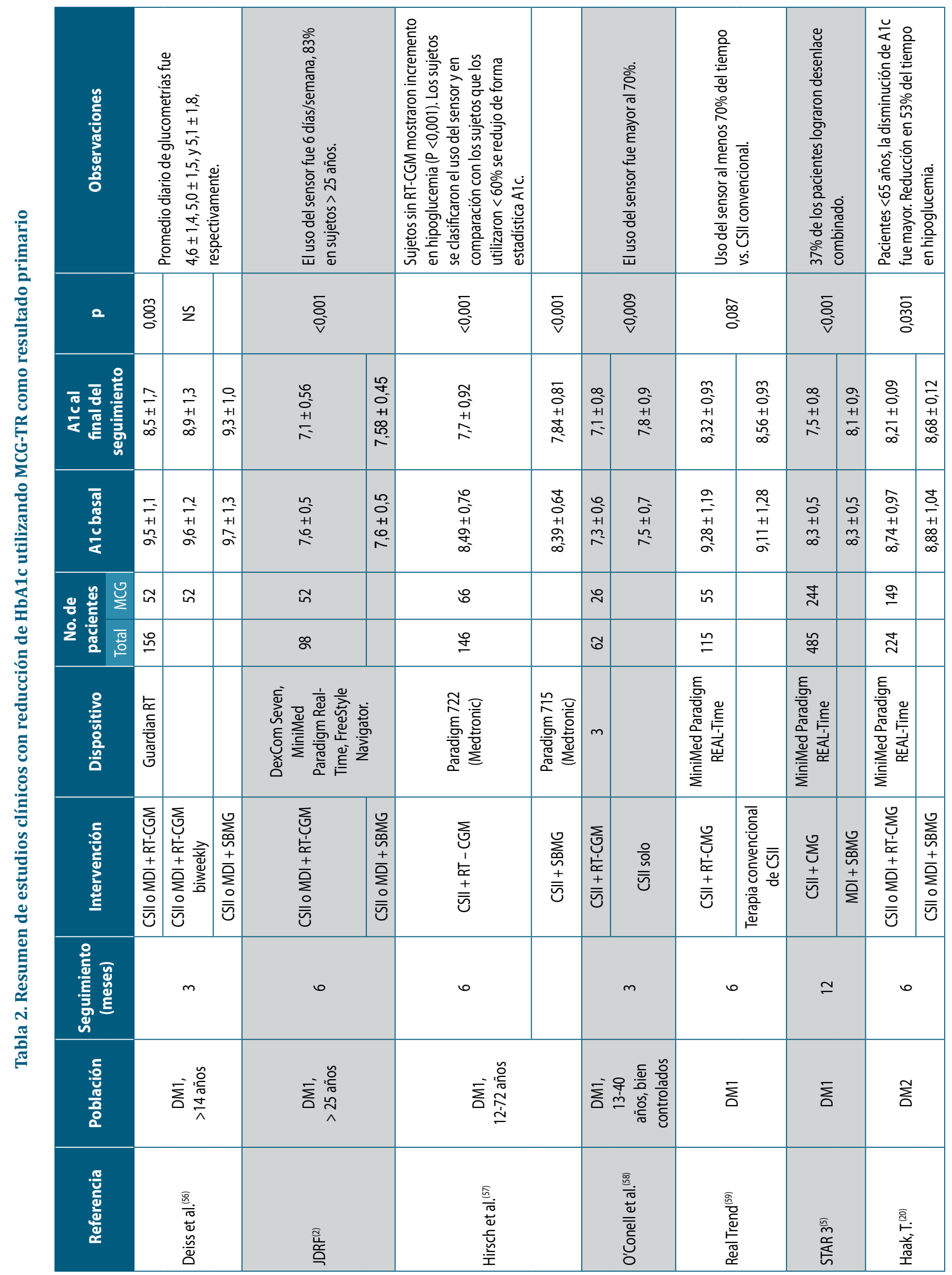




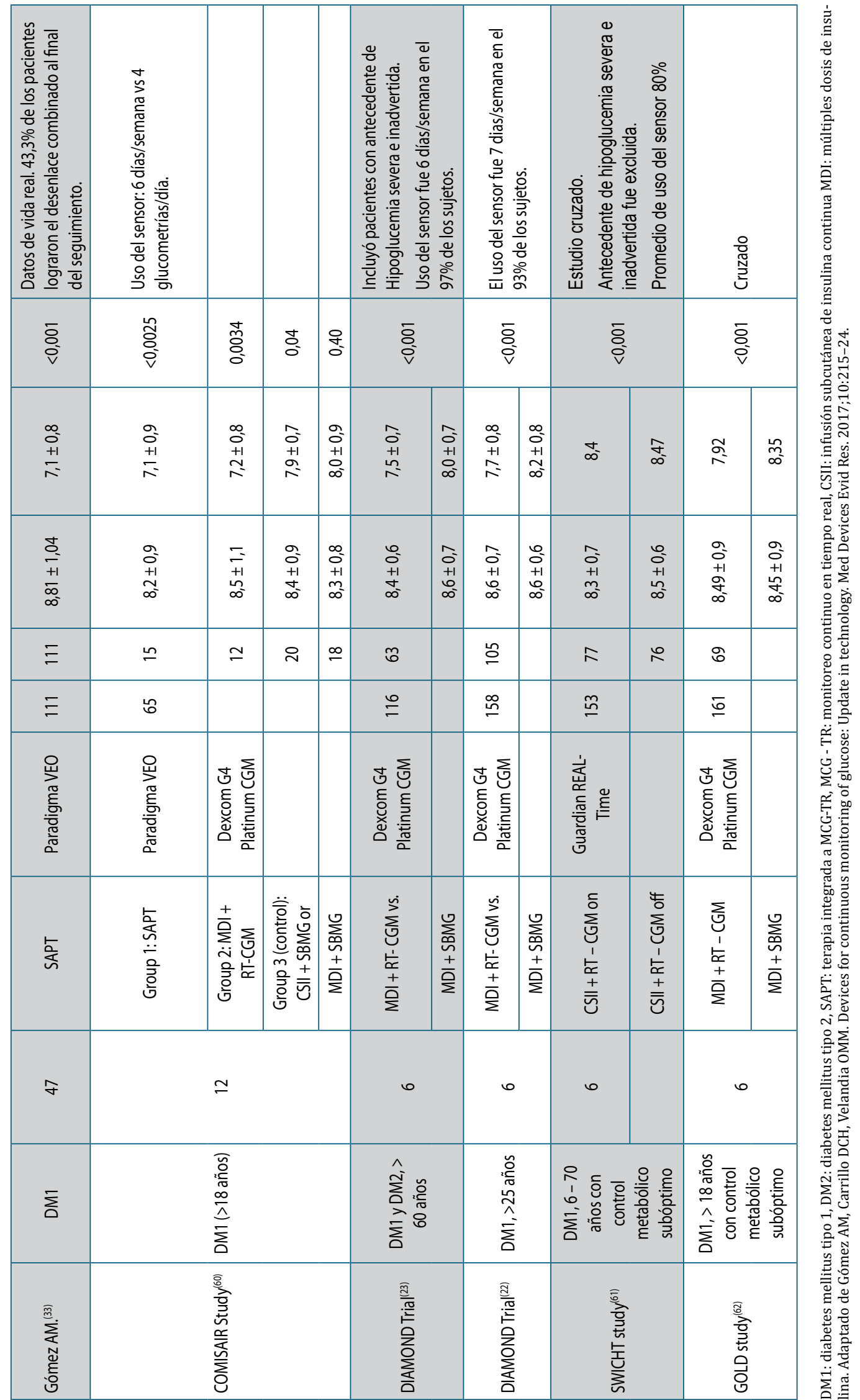


El Sistema Híbrido de Circuito Cerrado MiniMed 670G (Medtronic, Northridge, CA, USA) utiliza un algoritmo de circuito cerrado y sensor de cuarta generación. El uso de este dispositivo mostró disminución significativa de la HbA1c de 7,4 $\pm 0,9 \%$ a $6,9 \pm 0,6 \%(\mathrm{p}<0,001)$ y de la variabilidad glucémica medida por el coeficiente de variación de 0,38 a 0,35 ( $\mathrm{p}<0,001)$ comparado con los valores basales, sin casos de cetoacidosis diabética ni hipoglucemia severa ${ }^{(12)}$. Este dispositivo fue aprobado por la FDA en octubre de $2016^{(12)}$, actualmente no está disponible en Colombia.

En conclusión, la implementación del MCG-TR en la práctica clínica y el desarrollo de dispositivos integrados han dado paso al diseño de diferentes algoritmos que involucran el uso de alarmas y funciones de suspensión automática de infusión de insulina, permitiendo alcanzar las metas de HbA1c de forma segura.

\section{Seguridad: reducción de hipoglucemia}

En el consenso de la Asociación Americana de Diabetes (ADA), la hipoglucemia se define como un episodio de baja concentración de glucosa que expone al individuo a un daño potencial, y se clasifica en alerta de hipoglucemia $(<70 \mathrm{mg} / \mathrm{dL}$ ), hipoglucemia clínicamente significativa $(<54 \mathrm{ml} / \mathrm{dL})$ e hipoglucemia severa $^{(24)}$.

La hipoglucemia severa y clínicamente significativa es una complicación común y una de las principales barreras para lograr un control glucémico óptimo; por otro lado, se ha descrito su relación con fracturas, accidentes automovilísticos, deterioro cognitivo, incrementos en los costos del sistema de salud y el aumento de riesgo de muerte por causas cardiovasculares $^{(25-29)}$. Se han descrito varios factores relacionados con hipoglucemia, incluyendo la terapia intensiva con insulina, la cual triplica los episodios de hipoglucemia y aumenta los episodios de hipoglucemia severa (62 vs. 19 episodios/100 pacientesaño) comparada con la terapia convencional ${ }^{(2,12)}$.

Existen diferentes estrategias para evitar la hipoglucemia, entre las cuales se encuentran la educación estructurada del paciente, metas individualizadas para pacientes de alto riesgo, y el $\mathrm{SMBG}^{(30)}$. Estudios con MCG han demostrado reducción en la frecuencia y la duración de hipoglucemia clínicamente significativa $^{(30)}$.

En un estudio clínico multicéntrico, controlado en pacientes con DM1 con adecuado control metabólico, se demostró una reducción del 50\% del tiempo en rango de hipoglucemia clínicamente significativa ( $<55 \mathrm{mg} / \mathrm{dL})$, con una reducción en el número de mediciones de glucosa capilar de $5,5 \pm 2,0$ a 0,5 $\pm 0,7$ glucometrías/día, aunque no se reportaron cambios significativos en $\mathrm{HbA} 1 \mathrm{c}$ en pacientes usuarios de MCG-TR ${ }^{(31)}$. Un estudio similar realizado recientemente mostró una reducción significativa del tiempo en hipoglucemia y del número de episodios en el grupo de intervención comparado con SMBG a los seis meses de seguimiento ${ }^{(30)}$. Pacientes con DM2 con MDI o infusión de insulina tuvieron una reducción del 53\% de tiempo en hipoglucemia, disminución en el número de mediciones de glucometría capilar de 3,8 $\pm 1,4$ a 0,3 $\pm 0,7$ glucometrías al día ${ }^{(20)}$. Esa reducción clínicamente relevante de hipoglucemia sin depender de una función de alarma se relacionó con la adherencia al uso del sensor ( $>90 \%$ del tiempo) y la frecuencia de escaneo ${ }^{(30)}$.

El estudio ASPIRE demostró una reducción de área bajo la curva de $37,5 \%$ para hipoglucemia nocturna, de los episodios de hipoglucemia nocturna, sin aumento en los niveles de HbA1c en comparación al grupo control en pacientes con DM1 usuarios de SAPT con la función LGS encendida ${ }^{(32)}$. Estudios a largo plazo en población real con alto riesgo de hipoglucemia documentaron una reducción significativa en la incidencia de hipoglucemia sintomática e inadvertida presentada en forma temprana de $80,1 \%$ a $10,8 \%$ y de $66,6 \%$ a $2,7 \%$, respectivamente $(\mathrm{p}<0,001)^{(33)}$. Un estudio descriptivo que utilizó SAPT con función PLGM (predictive low glucose management) describió reducción del $80 \%$ de los eventos de hipoglucemia ${ }^{(34)}$.

Dos estudios clínicos aleatorizados recientes evaluaron el beneficio del MCG-TR comparado con SMBG en términos de reducción de hipoglucemia en pacientes con DM1 considerados en alto riesgo de hipoglucemia ya fuese por presentar hipoglucemias inadvertidas o hipoglucemias severas. El primero de ellos utilizó un diseño cruzado e incluyó pacientes tanto con MDI como con infusión continua de insulina (no integrada a MCG) y reportó una diferencia significativa en el número de eventos totales de hipoglucemia severa en la fase de SMBG versus MCG (34 versus 14 eventos, $p=0,033$ ), así como una diferencia significativa en el tiempo en rango menor o igual a 70 $\mathrm{mg} / \mathrm{dL}$ en las 24 horas y en el segmento nocturno de $-4,7 \%$ (95\% IC: $-5.9,-3.4, \mathrm{p}<0.001$ ) y - 5.7\% (95\% IC -8.2, -3.2, p< $0.0001)$ respectivamente, sin deterioro de la HbA1c ${ }^{(35)}$. El segundo estudio evaluó el impacto del MCG en pacientes con historia de hipoglucemias severas o no percibidas e incluyó únicamente pacientes con MDI, este demostró una disminución en el riesgo de hipoglucemia severa con el uso del MCG - TR y una disminución en el porcentaje de tiempo menor o igual de 70 y de $54 \mathrm{mg} / \mathrm{dL}$ por MCG, nuevamente sin un deterioro aso-

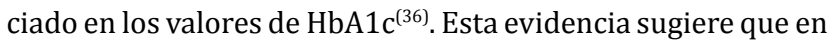
pacientes con DM1, en alto riesgo de hipoglucemia, el uso del MCG-TR, disminuye las métricas de hipoglucemia y los eventos de hipoglucemia severa, sin deteriorar el control metabólico.

\section{Reducción de variabilidad glucémica}

La variabilidad glicémica se define como las fluctuaciones en los niveles de glucosa en un periodo de tiempo establecido ${ }^{(37)}$, es un predictor de hipoglucemia independiente de la HbA1c y recientemente se describió su relación con mortalidad por cualquier causa $^{(29,38-41)}$. En el 2016 un estudio concluyó que la variabilidad glucémica, medida por el porcentaje de coeficiente de variación $(\% \mathrm{CV})$ con un punto de corte de $36 \%$, se asocia 
con mayor frecuencia de hipoglucemia ${ }^{(41)}$. Dada la importancia en establecer la mejor métrica para evaluar la variabilidad glucémica en Colombia se realizó un estudio de cohorte con 140 pacientes con diabetes tipo 2 y antecedente de hipoglucemia. Se encontró una fuerte asociación entre \%CV y otras medidas como DE $(r=0,84)$, CONGA $(r=0,84)$, IQR $(r=0,96)$ y MAGE ( $r$ $=0,96)$, adicionalmente, se estableció como punto de corte de \%CV 34\% como predictor de hipoglucemia ${ }^{(42)}$.

Aunque se pueden evaluar las fluctuaciones diarias de los niveles de glucosa con SMBG, la correlación con hipoglucemia es baja ${ }^{(43)}$. Con el desarrollo del MCG, estas fluctuaciones pueden ser evaluadas en escala de minutos con adecuada correlación con el riesgo de hipoglucemia ${ }^{(43)}$. Es importante utilizar el MCG en la práctica clínica diaria para detectar aquellos pacientes con variabilidad glucémica elevada, dada su asociación con hipoglucemia y teniendo en cuenta que actualmente disponemos de intervenciones que permiten reducir la variabilidad glucémica, entre ellos insulinas de acción ultra larga ${ }^{(44)}$, inhibidores de DPP4 ${ }^{(45)}$, análogos de GLP1 ${ }^{(46)}$, permitiendo ofrecer alternativas de tratamiento más seguras.

Dado que el MCG-TR permite la detección de la tendencia de los niveles de glucosa, alertando al paciente con alarmas para que tome las medidas necesarias para evitar tanto la hiper como la hipoglucemia, favorece la reducción de la variabilidad glucémica ${ }^{(12)}$. Estudios han descrito que el uso de MCGTR en MDI y en usuarios de sistemas de infusión continua de insulina reduce la variabilidad glucémica y reduce el tiempo de exposición a hipoglucemia ${ }^{(47,48)}$, sin embargo, se requieren estudios adicionales para confirmar esta hipótesis.

\section{Utilidad del MCG-TR en mujeres con diagnóstico de diabetes en embarazo}

Se encontraron dos estudios que han reportado desenlaces de control glucémico y materno-fetales asociados al uso del MCG-TR en mujeres embarazadas. Uno de ellos incluyó mujeres con diabetes mellitus tipo 1 y tipo 2 , y tenía como objetivo comparar el uso del MCG-TR versus SMBG (siete veces al día), con la anotación de que el MCG-TR se utilizó de manera intermitente por seis días en diferentes semanas del embarazo y era evaluado por el médico para la realización de ajustes en la terapia con insulina. En este estudio no se documentaron diferencias en el control glucémico ni en desenlaces fetales ${ }^{(49)}$. Sin embargo, un estudio multicéntrico aleatorizado que incluyó más de 300 mujeres con DM1, reclutadas en el primer trimestre del embarazo o durante la etapa prenatal y que fueron aleatorizadas a uso de MCG-TR de manera permanente o a SMBG (siete veces al día), demostró una mayor disminución, pequeña pero significativa, en los valores de HbA1c en la semana 24 y 34 con respecto a la línea de base a favor del grupo de MCG-TR (-0,67\% vs. $-0,52 \%, p=0,374$ y $-0,54 \%$ vs. $-0,35 \% p=0,372$, respectivamente), con un mayor tiempo en rango en la semana 34 en el grupo de MCG (68\% vs. $61 \% \mathrm{p}=0,0034)$ y menor tiempo en hiperglucemia $>140$ $\mathrm{mg} / \mathrm{dL}$ (27\% vs. 32\%, p=0,0279) en el grupo de MCG-TR, así como mejoría en algunos desenlaces fetales como macrosomía (53\% vs. $69 \%$ p=0,021), hipoglucemia neonatal $(15 \%$ vs. $28 \% \mathrm{p}=0,025$ ) y necesidad de cuidado intensivo neonatal durante más de 24 horas ( $27 \%$ vs. $43 \%$, p=0,0157), posiblemente atribuidos a menor tiempo de exposición en hiperglucemia en el grupo de MCG-TR ${ }^{(50)}$.

\section{Evidencia clínica y experiencia del uso de MCG-TR en Colombia}

Colombia es un país pionero en el uso de MCG en América Latina y actualmente dispone de varios centros especializados en el manejo de pacientes usuarios de esta tecnología. Un estudio realizado en Medellín describió una cohorte de 183 pacientes con diabetes mellitus tipo 1 y 2 con disminución significativa de niveles de $\mathrm{HbA} 1 \mathrm{C}$ de $8,7 \% \pm 1,7 \%$ y $7,4 \% \pm 0,8 \%$ $(\mathrm{P}<0,05)$, disminución en ingresos a hospitalización de 16,5\% a $6,0 \%(\mathrm{p}<0,05)$ y disminución de la incidencia de hipoglucemia severa $32 \%$ a $7,1 \%$ durante un año de seguimiento ${ }^{(51)}$. Datos a largo plazo muestran que el uso de la terapia SAP con la función LGS activada reduce los niveles de HbA1c de forma temprana, entre 3 y 5 meses posterior al inicio de la terapia y se mantiene por $47 \pm 22,7$ meses con disminución en los eventos de hipoglucemia y un aumento significativo en el porcentaje de la población que cumplía los criterios de desenlace compuesto, es decir, HbA1c menor o igual a 7\% en ausencia de hipoglucemia $2,7 \%$ a $42,3 \%(\mathrm{p}<0001)^{(33)}$. Hallazgos similares han sido descritos en otras series, sugiriendo que el MCG-TR es una herramienta útil que permite alcanzar metas estrictas de control metabólico, con una reducción en los episodios de hipoglucemia severa $^{(4,5,7,21)}$.

En 2017 se publicaron datos acerca de la experiencia con SAPT con función LGS activada en pacientes embarazadas con diabetes tipo 1, describiendo disminución significativa de HbA1c desde el primer trimestre de $8,19 \% \pm 0,83 \%$ a $7,16 \% \pm$ $0,92 \%(p=0,043)$, en el tercer trimestre del embarazo, el $66,6 \%$ de las pacientes lograron $\mathrm{HbA1c}$ menor que $6,5 \%$ con un significado clínico y estadísticamente significativo $(\mathrm{p}<0,001)^{(52)}$, hallazgos similares se describieron en la cohorte de Medellín ${ }^{(53)}$.

Datos preliminares de 54 pacientes con DM1 con una HbA1c base de 8,0 $\pm 1,3 \%$ a quienes se les inició SAPT con la función PLGM activada, a los tres meses de seguimiento muestran una disminución de la HbA1c a 7,34 $\pm 0,94$ ( $\mathrm{p}=0,0001)$, un descenso significativo de la incidencia de hipoglucemia severa de $1,11 \pm 2,05$ a $0,037 \pm 0,27$ episodios por paciente durante tres meses $(\mathrm{p}<0,0001)$, con una reducción significativa del área bajo la curva $<70 \mathrm{mg} / \mathrm{dL}$ de $1,39 \pm 3,14$ a 0,48 $\pm 0,63$ $(\mathrm{p}=0,048)^{(12)}$.

A pesar de la evidencia que respalda el uso de SAPT a ni- 
vel mundial y local, su costo ha dificultado la implementación en los diferentes sistemas de salud. En un estudio realizado en Colombia usando el CORE Diabetes Model, se documentó incremento en el promedio de expectativa de vida calculado en 3,51 años (IC 95\%: 3,47-3,55) y 3,81 años ajustados por calidad adicional, en pacientes con alto riesgo de hipoglucemia usuarios de SAPT comparado con MDI. Adicionalmente se retrasó entre 1,15 y 1,74 años la aparición de complicaciones relacionadas con pobre control metabólico, con un promedio de tiempo libre de complicaciones de 4,05 años. El tiempo promedio libre de neuropatía, proteinuria y retinopatía proliferativa fue de 4,5, 4,79 y 4,91 años, respectivamente; adicionalmente, la incidencia acumulada de enfermedad renal terminal fue de 10,1\% vs. 18,5\% comparada con MDI. Cuando se llevó a cabo el análisis de costo-efectividad, los costos derivados del uso de esta tecnología fueron parcialmente compensados a los ahorros atribuidos a la reducción de complicaciones microvasculares ${ }^{(54)}$, otros estudios han portado conclusiones similares $^{(55)}$. Las publicaciones de estos trabajos permitieron la inclusión de esta tecnología en sistemas de salud de países como Colombia, donde el costo de la terapia se encuentra totalmente cubierto.

\section{Limitaciones}

El MCG es una herramienta disponible en diversos países; sin embargo, existen ciertas limitaciones, entre ellas, la vida media relativamente corta del sensor y el requerimiento de glucometrías para calibración requerido por algunos dispositivos para asegurar la precisión del sensor. Adicionalmente, requiere personal capacitado en la interpretación y uso de los diferentes dispositivos disponibles, que en conjunto con el alto costo de esta tecnología han restringido su uso ${ }^{(9,12,30)}$.

\section{Conclusión}

Los dispositivos de MCG permiten a los clínicos evaluar el control glucémico, detectar hipoglucemias, especialmente nocturnas, y proveen información adicional, incluyendo la variabilidad glucémica. El desarrollo de terapias integradas posibilita el cumplimiento de metas de control glucémico estrictas con una disminución en los episodios de hipoglucemia severa en una población de alto riesgo. Aunque el costo de la terapia es una de las principales limitaciones para su uso, la SAPT ha demostrado ser costo-efectiva en diversos países incluyendo a Colombia, en términos de incremento de expectativa de vida, retraso de aparición y progresión de complicaciones microvasculares, con una reducción de costos en términos de episodios de hipoglucemia severa, ofreciendo así una alternativa segura en el tratamiento de pacientes diabéticos. No obstante, se necesita impulsar la diseminación de implementación de esta tecnología, el desarrollo de sensores más baratos y precisos, y la familiarización de los médicos con las ventajas del uso de esta tecnología.

\section{Declaración de conflictos}

Los autores declaran los siguientes potenciales conflictos de interés con respecto a la investigación, autoría y/o publicación del presente artículo: AMG reporta honorarios de orador de Novo Nordisk, Elli Lilly, MSD, Novartis y Medtronic; financiación para investigación de Medtronic, Novartis, Novo Nordisk y Abbott. DCH reporta honorarios de orador y financiación de investigación de Novo Nordisk y Medtronic. No se reportan otros posibles conflictos.

\section{Agradecimientos}

Estamos agradecidos con nuestro equipo de trabajo del Centro de Diabetes en el Hospital Universitario San Ignacio y el apoyo de la Asociación Colombiana de Endocrinología, Diabetes y Metabolismo. 


\section{Referencias}

1. Group UPDS (UKPDS). blood-glucose control with sulphonylureas or insulin compared with conventional treatment and risk of complications in patients with type 2 diabetes (UKPDS 33). Lancet [Internet]. 1998;352(9131):83753. Available from: http://discovery.ucl.ac.uk/1310755/

2. Nathan DM, Genuth S, Lachin J, Cleary P, Crofford O, Davis M, et al. The effect of intensive treatment of diabetes on the development and progression of long-term complications in insulin-dependent diabetes mellitus. N Engl J Med. 1993 Sep;329(14):977-86.

3. Danne T, Nimri R, Battelino T, Bergenstal RM, Close KL, DeVries JH, et al. International consensus on use of continuous glucose monitoring. Diabetes Care. 2017;40(12):1631-40.

4. Hoeks LBEA, Greven WL, de Valk HW. Real-time continuous glucose monitoring system for treatment of diabetes: A systematic review. Diabet Med. 2011;28(4):386-94

5. Claus Vogelmeier, M.D., Bettina Hederer, M.D., Thomas Glaab, M.D., Hendrik Schmidt PD, Maureen P.M.H. Rutten-van Mölken, Ph.D., Kai M. Beeh, M.D. Klaus F. Rabe, M.D., and Leonardo M. Fabbri MD, Investigators for the P-C. New England Journal. Clin Trials. 2010;363:411-22.

6. Pickup JC, Freeman SC, Sutton AJ. Glycaemic control in type 1 diabetes during real time continuous glucose monitoring compared with self monitoring of blood glucose: Meta-analysis of randomised controlled trials using individual patient data. Bmj. 2011;343(7815).

7. Gómez Medina AM, Marín Sánchez A. Monitorización continua de glucosa en tiempo real: Imprescindible su uso combinado con infusión continua de insulina. Av en Diabetol. 2011;27(4):143-50.

8. Bruttomesso D. Technological Advances in the Treatment of Type 1 Diabetes. 2014. 81-98 p.

9. Rodbard D. Continuous Glucose Monitoring: A Review of Successes, Challenges, and Opportunities. Diabetes Technol Ther [Internet]. 2016;18(S2):S2-3-S2-13. Available from: http://online.liebertpub.com/ doi/10.1089/dia.2015.0417

10. Keenan DB, Mastrototaro JJ, Voskanyan G, Steil GM. Delays in minimally invasive continuous glucose monitoring devices: A review of current technology. J Diabetes Sci Technol. 2009;3(5):1207-14.

11. Kubiak T, Hermanns N, Schreckling HJ, Kulzer B, Haak T. Assessment of hypoglycaemia awareness using continuous glucose monitoring. Diabet Med. 2004;21(5):487-90.

12. Gómez AM, Carrillo DCH, Velandia OMM. Devices for continuous monitoring of glucose: Update in technology. Med Devices Evid Res. 2017;10:215-24.

13. Gómez AM, Marín Sánchez A, Muñoz OM, Colón Peña CA. Precisión numérica y clínica de la monitorización continua de glucosa en pacientes colombianos en terapia con bomba de infusión de insulina con suspensión automática en hipoglucemia. Endocrinol y Nutr. 2015;62(10):485-92.

14. Pfützner A, Klonoff DC, Pardo S, Parkes JL. Technical aspects of the Parkes error grid. J Diabetes Sci Technol. 2013;7(5):1275-81.

15. Bailey TS, Grunberger G, Bode BW, Handelsman Y, Hirsch IB, Jovanovič L, et al. American Association of Clinical Endocrinologists and American College of Endocrinology 2016 Outpatient Glucose Monitoring Consensus Statement. Endocr Pract [Internet]. 2016;22(2):231-61. Available from: http:// journals.aace.com/doi/10.4158/EP151124.CS

16. Aberer F, Hajnsek M, Rumpler M, Zenz S, Baumann PM, Elsayed H, et al. Evaluation of subcutaneous glucose monitoring systems under routine environmental conditions in patients with type 1 diabetes. Diabetes, Obes Metab. 2017;19(7):1051-5.

17. Kropff J, DeVries JH. Continuous Glucose Monitoring, Future Products, and Update on Worldwide Artificial Pancreas Projects. Diabetes Technol Ther [Internet]. 2016;18(S2):S2-53-S2-63. Available from: http://online.liebertpub.com/doi/10.1089/dia.2015.0345

18. Vaddiraju S, Burgess DJ, Tomazos I, Jain FC, Papadimitrakopoulos F. Technologies for continuous glucose monitoring: Current problems and future promises. J Diabetes Sci Technol. 2010;4(6):1540-62.

19. Bailey T, Bode BW, Christiansen MP, Klaff LJ, Alva S. The Performance and Usability of a Factory-Calibrated Flash Glucose Monitoring System. Diabetes Technol Ther [Internet]. 2015;17(11):787-94. Available from: http:// online.liebertpub.com/doi/pdfplus/10.1089/dia.2014.0378

20. Haak T, Hanaire H, Ajjan R, Hermanns N, Riveline JP, Rayman G. Flash Glucose-Sensing Technology as a Replacement for Blood Glucose Monitoring for the Management of Insulin-Treated Type 2 Diabetes: a Multicenter Open-Label Randomized Controlled Trial. Diabetes Ther. 2017;8(1):55-73.

21. Juvenille Diabetes Research Foundation Continuous Glucose Monitoring Study Group. Continuous glucose monitoring and intensive treatment of type 1 diabetes. N Engl J. [Internet]. 2008;359(14):1464-76. Available from: http://www.ncbi.nlm.nih.gov/m/pubmed/18779236/\%5Cnhttp://www. ncbi.nlm.nih.gov/pubmed/18779236

22. Beck RW, Riddlesworth T, Ruedy K, Ahmann A, Bergenstal R, Haller S, et al. Effect of Continuous Glucose Monitoring on Glycemic Control in Adults With Type 1 Diabetes Using Insulin Injections. Jama [Internet]. 2017;317(4):371. Available from: http://jama.jamanetwork.com/article.aspx?doi=10.1001/ jama.2016.19975
23. Ruedy KJ, Parkin CG, Riddlesworth TD, Graham C. Continuous Glucose Monitoring in Older Adults With Type 1 and Type 2 Diabetes Using Multiple Daily Injections of Insulin: Results From the DIAMOND Trial. J Diabetes Sci Technol. 2017;11(6):1138-46.

24. Riddle MC, Bakris G, Blonde L, Boulton AJM, D 'alessio D, De Groot M, et al Introduction: Standards of Medical Care in Diabetes-2018. Diabetes Care [Internet]. 2018;41(Supplement 1):S1-2. Available from: http://care.diabetesjournals.org/lookup/doi/10.2337/dc18-Sint01

25. Conceição J, Dores J, Araújo F, Laires PA, Carr RD, Brodovicz K, et al. Severe hypoglycaemia among patients with type 2 diabetes requiring emergency hospital admission: The Hypoglycaemia In Portugal Observational StudyEmergency Room (HIPOS-ER). Diabetes, Obes Metab. 2018;20(1):50-9.

26. Hung YC, Lin CC, Chen HJ, Chang MP, Huang KC, Chen YH, et al. Severe hypoglycemia and hip fracture in patients with type 2 diabetes: a nationwide population-based cohort study. Osteoporos Int. 2017;28(7):2053-60.

27. Aronson R, Goldenberg R, Boras D, Skovgaard R, Bajaj H. The Canadian Hypoglycemia Assessment Tool Program: Insights Into Rates and Implications of Hypoglycemia From an Observational Study. Can J Diabetes [Internet]. 2016;1-7. Available from: http://dx.doi.org/10.1016/j.jcjd.2017.01.007

28. Lee AK, Warren B, Lee CJ, Mcevoy JW, Matsushita K, Huang ES, et al. The Association of Severe Hypoglycemia With Incident Cardiovascular Events and Mortality in Adults With Type 2 Diabetes. 2017;(7):1-8.

29. Pieber TR, Marso SP, McGuire DK, Zinman B, Poulter NR, Emerson SS, et al DEVOTE 3: temporal relationships between severe hypoglycaemia, cardiovascular outcomes and mortality. Diabetologia. 2018;61(1):58-65.

30. Bolinder J, Antuna R, Geelhoed-Duijvestijn P, Kröger J, Weitgasser R Novel glucose-sensing technology and hypoglycaemia in type 1 diabetes: a multicentre, non-masked, randomised controlled trial. Lancet. 2016;388(10057):2254-63.

31. Stephan Matthaei. An Evaluation of Novel Glucose Sensing Technology on Hypoglycaemia in Type 1 Diabetes (IMPACT) [Internet]. NCT02232698. 2017 [cited 2017 May 29]. Available from: https://clinicaltrials.gov/ct2/ show/NCT02232698

32. Bergenstal RM, Klonoff DC, Garg SK, Bode BW, Meredith M, Slover RH, et al. Threshold-Based Insulin-Pump Interruption for Reduction of Hypoglycemia. N Engl J Med [Internet]. 2013;369(3):224-32. Available from: http:// www.nejm.org/doi/10.1056/NEJMoa1303576

33. Gómez AM, Marín Carrillo LF, Muñoz Velandia OM, Rondón Sepúlveda MA Arévalo Correa CM, Mora Garzón E, et al. Long-Term Efficacy and Safety of Sensor Augmented Insulin Pump Therapy with Low-Glucose Suspend Feature in Patients with Type 1 Diabetes. Diabetes Technol Ther [Internet]. 2016;19(2):dia.2016.0332. Available from: http://online.liebertpub.com/ doi/10.1089/dia.2016.0332

34. Choudhary P, Olsen BS, Conget I, Welsh JB, Vorrink L, Shin JJ. Hypoglycemia Prevention and User Acceptance of an Insulin Pump System with Predictive Low Glucose Management. Diabetes Technol Ther [Internet]. 2016;18(5):288-91. Available from: http://online.liebertpub.com/ doi/10.1089/dia.2015.0324

35. van Beers CAJ, DeVries JH, Kleijer SJ, Smits MM, Geelhoed-Duijvestijn PH, Kramer $\mathrm{MHH}$, et al. Continuous glucose monitoring for patients with type 1 diabetes and impaired awareness of hypoglycaemia (IN CONTROL): a randomised, open-label, crossover trial. Lancet Diabetes Endocrinol. 2016;4(11):893-902.

36. Heinemann L, Freckmann G, Ehrmann D, Faber-Heinemann G, Guerra S, Waldenmaier D, et al. Real-time continuous glucose monitoring in adults with type 1 diabetes and impaired hypoglycaemia awareness or severe hypoglycaemia treated with multiple daily insulin injections (HypoDE): A multicentre, randomised controlled trial. Lancet [Internet]. 2018;6736(18):111. Available from: http://dx.doi.org/10.1016/S0140-6736(18)30297-6

37. Monnier L, Colette C, Owens DR. The application of simple metrics in the assessment of glycaemic variability. Diabetes Metab [Internet]. 2018;1-7. Available from: https://doi.org/10.1016/j.diabet.2018.02.008

38. Kramer CK, Choi H, Zinman B, Retnakaran R. Glycemic variability in patients with early type 2 diabetes: The impact of improvement in ??-cell function. Diabetes Care. 2014;37(4):1116-23.

39. Kilpatrick ES, Rigby AS, Goode K, Atkin SL. Relating mean blood glucose and glucose variability to the risk of multiple episodes of hypoglycaemia in type 1 diabetes. Diabetologia. 2007;50(12):2553-61.

40. Johnson SL, McEwen LN, Newton CA, Martin CL, Raskin P, Halter JB, et al. The impact of continuous subcutaneous insulin infusion and multiple daily injections of insulin on glucose variability in older adults with type 2 diabetes. J Diabetes Complications. 2011;25(4):211-5.

41. Monnier L, Colette C, Wojtusciszyn A, Dejager S, Renard E, Molinari N, et al Toward Defining the Threshold Between Low and High Glucose Variability in Diabetes. Diabetes Care [Internet]. 2016;dc161769. Available from: http://care.diabetesjournals.org/lookup/doi/10.2337/dc16-1769

42. Gómez AM, Muñoz OM, Marin A, Fonseca MC, Rondon M, Robledo Gómez MA, et al. Different Indexes of Glycemic Variability as Identifiers of Patients with Risk of Hypoglycemia in Type 2 Diabetes Mellitus. J Diabetes Sci Technol [Internet]. 2018;(40):193229681875810. Available from: http://journals.sagepub.com/doi/10.1177/1932296818758105 
43. Kovatchev B, Cobelli C. Glucose variability: Timing, risk analysis, and relationship to hypoglycemia in diabetes. Diabetes Care. 2016;39(4):502-10.

44. Henao-Carrillo DC, Muñoz OM, Gómez AM, Rondón M, Colón C, Chica L, et al. Reduction of glycemic variability with Degludec insulin in patients with unstable diabetes. J Clin Transl Endocrinol [Internet]. 2018;12(April):8-12. Available from: https://www.sciencedirect.com/science/article/pii/ S2214623718300206

45. Guerci B, Monnier L, Serusclat P, Petit C, Valensi P, Huet D, et al. Continuous glucose profiles with vildagliptin versus sitagliptin in add-on to metformin: Results from the randomized Optima study. Diabetes Metab [Internet]. 2012;38(4):359-66. Available from: http://dx.doi.org/10.1016/j. diabet.2012.06.001

46. Lane, W., Weinrib, J., Hale C. The effect of addition of liraglutide to high-dose intensive insulin therapy: a randomized prospective trial. Diabetes, Obes Andm. 2014;6-11.

47. Jamiołkowska M, Jamiołkowska I, Łuczyński W, Tołwińska J, Bossowski A, Głowińska Olszewska B. Impact of Real-Time Continuous Glucose Monitoring Use on Glucose Variability and Endothelial Function in Adolescents with Type 1 Diabetes: New Technology-New Possibility to Decrease Cardiovascular Risk? J Diabetes Res [Internet]. 2016;2016:1-8. Available from: http://www.hindawi.com/journals/jdr/2016/4385312/

48. Tumminia A, Crimi S, Sciacca L, Buscema M, Frittitta L, Squatrito S, et al. Efficacy of real-time continuous glucose monitoring on glycaemic control and glucose variability in type 1 diabetic patients treated with either insulin pumps or multiple insulin injection therapy: A randomized controlled crossover trial. Diabetes Metab Res Rev. 2015;31(1):61-8.

49. Secher A, Ringholm L, Andersen H, Damm P, Mathiesen E. The Effect of RealTime Continuous Glucose Monitoring in Pregnant Women With Diabetes. Diabetes Care. 2013;36:1877.

50. Feig DS, Donovan LE, Corcoy R, Murphy KE, Amiel SA, Hunt KF, et al. Continuous glucose monitoring in pregnant women with type 1 diabetes (CONCEPTT): a multicentre international randomised controlled trial. Lancet. 2017;390(10110):2347-59.

51. Ramirez-Rincon A, Hincapie-García J, Arango CM, Aristizabal N, Castillo E, Hincapie G, et al. Clinical Outcomes After 1 Year of Augmented Insulin Pump Therapy in Patients with Diabetes in a Specialized Diabetes Center in Medellín, Colombia. Diabetes Technol Ther [Internet]. 2016;18(11):713-8. Available from: http://onlineliebertpub.com/doi/10.1089/dia.2016.0166

52. Gómez AM, Marín Carrillo LF, Arévalo Correa CM, Muñoz Velandia OM, Rondón Sepúlveda MA, Silva Herrera JL, et al. Maternal-Fetal Outcomes in 34 Pregnant Women with Type 1 Diabetes in Sensor-Augmented Insulin Pump Therapy. Diabetes Technol Ther [Internet]. 2017;19(7):417-22. Available from: http://online.liebertpub.com/doi/10.1089/dia.2017.0030

53. Restrepo-Moreno M, Ramirez-Rincon A, Hincapie-Garcia J, Palacio A, Monsalve-Arango C, Aristizabal-Henao N, et al. Maternal and perinatal outcomes in pregnant women with type 1 diabetes treated with continuous subcutaneous insulin infusion and real time continuous glucose monitoring in two specialized centers in Medellin, Colombia. J Matern Fetal Neonatal Med. 2018 Mar;31(6):696-700.

54. Quitian H, Gomez A, Garcia Peña A, Arciniegas J, Iragorri N, Mantilla B, et al. Cost-Utility Analysis of Continuous Infusion Pump With Integrated Monitoring Compared With Multiple Daily Injection Treatment for Patients 15-Years or Older With Type 1 Diabetes Mellitus In Colombia. Value Hea [Internet]. 2015;18(7):A360. Available from: http://linkinghub.elsevier. com/retrieve/pii/S1098301515027692

55. Roze S, Saunders R, Brandt AS, de Portu S, Papo NL, Jendle J. Health-economic analysis of real-time continuous glucose monitoring in people with Type 1 diabetes. Diabet Med. 2015;32(5):618-26.

56. Deiss D, Bolinder J, Riveline JP, Battelino T, Bosi E, Tubiana-Rufi N, et al. Improved glycemic control in poorly controlled patients with type 1 diabetes using real-time continuous glucose monitoring. Diabetes Care. 2006;29(12):2730-2.

57. Hirsch IB, Abelseth J, Bode BW, Fischer JS, Kaufman FR, Mastrototaro J, et al Sensor-Augmented Insulin Pump Therapy: Results of the First Randomized Treat-to-Target Study. Diabetes Technol Ther [Internet]. 2008;10(5):37783. Available from: http://online.liebertpub.com/doi/abs/10.1089/ dia.2008.0068

58. O'Connell MA, Donath S, O'Neal DN, Colman PG, Ambler GR, Jones TW, et al Glycaemic impact of patient-led use of sensor-guided pump therapy in type 1 diabetes: A randomised controlled trial. Diabetologia. 2009;52(7):1250-7.

59. Denis $R$, Véronique $S$, Yves $R$, Bruno $G$, Eric $R$, Hélène $H$, et al. 11-Incremental Value of Continuous Glucose Monitoring When Starting Pump Therapy in Patients With Poorly Controlled Type 1 Diabetes. Diabetes Care. 2009;32(12):2245-2250.

60. Šoupal J, Petruželková L, Flekač M, Pelcl T, Matoulek M, Daňková M, et al Comparison of Different Treatment Modalities for Type 1 Diabetes, Including Sensor-Augmented Insulin Regimens, in 52 Weeks of Follow-Up: A COMISAIR Study. Diabetes Technol Ther [Internet]. 2016;18(9):532-8. Available from: http://online.liebertpub.com/doi/10.1089/dia.2016.0171

61. Conget I, Battelino T, Giménez M, Gough H, Castañeda J, Bolinder J. The SWITCH Study (Sensing With Insulin pump Therapy to Control $\mathrm{HbA}_{1 c}$ ): Design and Methods of a Randomized Controlled Crossover Trial on Sensor-Augmented Insulin Pump Efficacy in Type 1 Diabetes Suboptimally Controlled with Pump Therapy. Diabetes Technol Ther [Internet] 2011;13(1):49-54. Available from: http://online.liebertpub.com/doi/ abs/10.1089/dia.2010.0107

62. Lind M, Polonsky W, Hirsch IB, Heise T, Bolinder J, Dahlqvist S, et al. Continuous glucose monitoring vs conventional therapy for glycemic control in adults with type 1 diabetes treated with multiple daily insulin injections the gold randomized clinical trial. JAMA - J Am Med Assoc. 2017;317(4):379-87. 